\title{
Consistent ICP for the registration of sparse and inhomogeneous point clouds
}

\author{
Hiêp Quang Luong*†, Michiel Vlaminck*, Werner Goeman ${ }^{\ddagger}$ and Wilfried Philips* \\ *Image Processing and Interpretation research group \\ Department of Telecommunications and Information Processing \\ Ghent University - iMinds, Ghent, Belgium \\ $\dagger$ Email: hiep.luong@UGent.be \\ $\ddagger_{\text {Grontmij, Ghent, Belgium }}$
}

\begin{abstract}
In this paper, we derive a novel iterative closest point (ICP) technique that performs point cloud alignment in a robust and consistent way. Traditional ICP techniques minimize the point-to-point distances, which are successful when point clouds contain no noise or clutter and moreover are dense and more or less uniformly sampled. In the other case, it is better to employ point-to-plane or other metrics to locally approximate the surface of the objects. However, the point-to-plane metric does not yield a symmetric solution, i.e. the estimated transformation of point cloud $p$ to point cloud $q$ is not necessarily equal to the inverse transformation of point cloud $q$ to point cloud $p$. In order to improve ICP, we will enforce such symmetry constraints as prior knowledge and make it also robust to noise and clutter. Experimental results show that our method is indeed much more consistent and accurate in presence of noise and clutter compared to existing ICP algorithms.
\end{abstract}

\section{INTRODUCTION}

The registration or alignment of point clouds is an important process in 3D mobile mapping, which is the process of collecting geospatial data from a mobile vehicle. In general, mobile mapping employs a wide range of different sensors, which can roughly be divided in two categories: the measurement sensors (e.g. 360-degrees vision cameras and LiDAR scanners) and positioning sensors (e.g. global positioning system (GPS), wheel encoders and inertial navigation system (INS)). GPS and inertial measurement units (IMU) allow for rapid and quite accurate determination of the position and attitude of the equipment or vehicle, or in other words, estimating the ego-localization. Still there are some shortcomings: e.g. GPS relies on external communication and lacks accuracy, while sequential IMU data (e.g. from gyroscopes and accelerometers) suffers from drift and must therefore be processed, e.g. by Kalman filters [5].

An alternative approach to ego-localization (when GPS and IMU are missing or unavailable) is to perform relative positioning based on images or overlapping point clouds. The class of algorithms that jointly estimates ego-localization (or odometry when we observe the positions over time) and the $3 \mathrm{D}$ reconstruction/mapping is called simultaneous localization and mapping (SLAM). The performance of visual SLAM (based on images or video) heavily depend on the environment such as weather and light conditions (e.g. overexposure due to direct sunlight). On the other hand, LiDAR technology is more robust to these conditions. A very popular and simple technique that performs LiDAR odometry and mapping is iterative closest point (ICP). ICP is the main component in large-scale 3D mapping and often it is combined with other techniques such as global pose graph optimization or loop closure in a larger framework to minimize drift and to enforce consistency on a global scale. In this paper, we will concentrate on improving the accuracy and consistency of the core ICP algorithm, which then can be plugged in large-scale 3D mapping frameworks.

LiDAR technology measures the distance between the objects and the device by illuminating the target with a laser light. The main advantage of LiDAR is that it can provide a lot of range measurements within small time window with errors that are relatively constant irrespective of the distances measured. An example of LiDAR scanners is the Velodyne series $^{1}$, which produce enormous point clouds, capturing the scene at approximately $10 \mathrm{~Hz}$ using a relative low number of scan lines (typically 16, 32 or 64 lines). Each full rotation or sweep produces an inhomogeneous point cloud that is densely sampled along the scan lines, but is sparse in the direction across the scan lines. This type of point clouds puts additional challenges in the alignment process, especially ICP: the measured points in two subsequent moved point clouds are (almost) never physically the same, which is a different starting point compared to the basic definition of ICP, which effectively minimizes the distances of the closest points assuming that they are physically the same. By locally approximating the surface of the scanned objects and minimizing the distance between a point and this surface, the registration is improved greatly.

With the emergence of affordable LiDAR technology, mobile mapping systems are evolved into the third generation. These new scanners become more lightweight, smaller and cheaper, which make them more attractive to be mounted on (aerial) drones or to be used in hand-held devices. We refer the interested reader to an extended review on existing mobile mapping technology [17] and overviews of commercially available mobile mapping systems, e.g. [14] and [22].

In the next sections, we briefly discuss the state-of-the-art

\footnotetext{
${ }^{1} \mathrm{http}: / /$ velodynelidar.com
} 
of related work and the concept of consistent registration, we propose a new consistent and robust ICP algorithm, we show some experiment results and we end this paper with a conclusion.

\section{RELATED WORK}

Point cloud registration is one of the important research issues in $3 \mathrm{D}$ imaging and computer vision due to its wide applications. The most often cited ICP algorithm is the traditional point-to-point ICP algorithm from Besl and McKay [2]. The key concept of the traditional ICP algorithm is estimating the transformation by iteratively computing correspondences between two point clouds and resolving the transformation that minimizes the distance between the corresponding points. Finding the correspondences is usually based on the Euclidean distance, which can be done efficiently for large point clouds using kd-trees. Zhang proposes to perform outlier rejection in this correspondence selection phase to make ICP more robust to errors [23]. Given two point clouds $\mathbf{p}$ and $\mathbf{q}$, we denote the set of corresponding points of $\mathbf{q}$ to $\mathbf{p}$ as $\mathbf{q}^{\prime}$. The estimation of the transformation $T_{\mathbf{p q}}$ from $\mathbf{p}$ to $\mathbf{q}$ (throughout this paper, we assume a 6-DOF model) can be found by minimizing the point-to-point metric:

$$
\hat{T}_{\mathbf{p q}}=\underset{T_{\mathbf{p q}}}{\arg \min } \sum_{i=1}^{N_{\mathbf{p}}}\left\|T_{\mathbf{p q}} \mathbf{p}_{i}-\mathbf{q}_{i}^{\prime}\right\|^{2},
$$

where $N_{\mathbf{p}}$ denotes the number of points in $\mathbf{p} ; \mathbf{p}_{i}$ and $\mathbf{q}_{i}^{\prime}$ are respectively the $i$ th point in $\mathbf{p}$ and $\mathbf{q}^{\prime}$. For reasons mentioned in the previous section, it is not preferred to employ the pointto-point metric for sparse and inhomogeneous point clouds. Chen and Medioni introduces the point-to-plane metric that matches points to (planar) surface patches, which are rough approximations of the object's surface [3]. The distance is now minimized along the surface normal while not penalizing offsets along the surface, resulting in the following optimization problem:

$$
\hat{T}_{\mathbf{p q}}=\underset{T_{\mathbf{p q}}}{\arg \min } \sum_{i=1}^{N_{\mathbf{p}}}\left\|\left(T_{\mathbf{p q}} \mathbf{p}_{i}-\mathbf{q}_{i}^{\prime}\right) \cdot \mathbf{n}_{\mathbf{q}_{i}^{\prime}}\right\|^{2},
$$

where $\mathbf{n}_{\mathbf{q}_{i}^{\prime}}$ is the normal estimated for point $\mathbf{q}_{i}^{\prime}$. Segal et al. combine the point-to-plane ICP algorithm into a probabilistic framework, in which the locally planar surface structure of both $\mathbf{p}$ and $\mathbf{q}$ is modeled [18]. This leads to a generalized plane-to-plane concept of ICP, making the problem more symmetrical and robust to noise and outliers.

Many other variants on ICP concentrate on acceleration (e.g. [11]), robustness (e.g. [7]), affine transformation (e.g. [4]), etc. We refer the interested reader to more complete overviews (e.g. [12], [15], [16], [19]) of point cloud registration using ICP.

As discussed in the previous section, ICP plays an important role in many LiDAR SLAM systems. Improvements to the ICP algorithm can directly be applied in such SLAM systems. A complete SLAM algorithm normally involves more than only
ICP: pre-processing and filtering, feature extraction, deskewing, drift correction, loop closure detection, incorporation of semantics about the environment or other data such as visual SLAM or positioning information, etc.(e.g. [13], [24], [25]).

\section{CONSISTENT REGISTRATION}

If we take a closer look at Equation (2), we see that it is not symmetric in $\mathbf{p}$ and $\mathbf{q}^{\prime}$. This means that the transformation from $\mathbf{p}$ to $\mathbf{q}$ is not necessarily equal to the inverse transformation from $\mathbf{q}$ to $\mathbf{p}$ (i.e. $T_{\mathbf{p q}} \neq T_{\mathbf{q p}}^{-1}$ ). As a result, the registration method may produce inconsistent results when applied in one direction versus the other.

In SLAM applications, ICP is computed for pairwise registrations, i.e. between two sweeps. This can be done successively, or with (semi-)fixed reference sweeps. Due to inaccuracies in pairwise registrations, the registration errors accumulate over time such that the registration parameters between the first and the last sweep can have a large cumulative error, which is also known as drift. This problem leads also to possible deadlocks, where it is impossible to find a proper global reconstruction when there is a loop in the trajectory. The deadlock problem can be solved using for example loop closure or global pose graph optimization techniques [20].

The asymmetry of the point-to-plane ICP solution and the deadlock problem indicate that there is a need for more consistent ICP algorithms. In literature, there are two common ways to achieve consistent registration, which are known as bundle adjustment and global registration. The latter solves all pairwise registrations jointly, which is often not desirable due to high computation and memory requirements. Bundle adjustment is a well-known computationally expensive tool in the computer vision community, which produces jointly optimal 3D structures and viewing parameters [21]. For sparse point clouds, this strategy is not optimal since the 3D points from different sweeps are physically different.

Related to global pose graph optimization, we can define loops on a very local scale, for example, the transformation from $\mathbf{p}$ to $\mathbf{q}$ should be equal to the inverse transformation from $\mathbf{q}$ to $\mathbf{p}$ (i.e. $T_{\mathbf{p q}} \equiv T_{\mathbf{q p}}^{-1}$ ). With an additional point cloud $\mathbf{r}$, we can assume that consecutive transformations from sweep $\mathbf{p}$ to $\mathbf{r}, \mathbf{r}$ to $\mathbf{q}$ and $\mathbf{q}$ to $\mathbf{p}$ should result in the identity transformation $I$ (i.e. $T_{\mathbf{q p}} T_{\mathbf{r q}} T_{\mathbf{p r}} \equiv I$ ). The set of combined pairwise transformations is conveniently described by the group structures of the Lie algebra [10]. We will employ this strategy to enforce pairwise transformations (and its parameters) to be in a valid Lie group structure and obtain as such consistent transformation parameters. Several group structures are illustrated in Figure 1. The aforementioned two examples coincide to the skew anti-symmetry and the Jacobi identity properties respectively.

In [6], the authors incorporated the consistency constraints as prior knowledge in a Bayesian framework of the gradientbased image registration for image super-resolution application. Instead of posing consistency with hard constraints, they penalized inconsistencies directly in the estimation problem of 


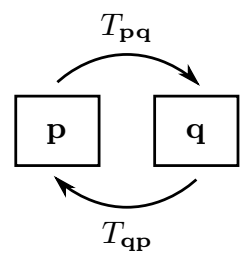

(a)

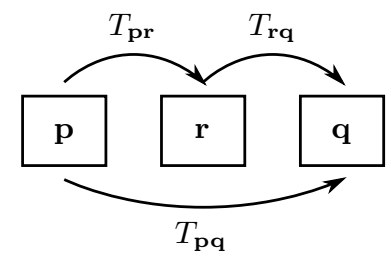

(b)
Fig. 1. Group structures in the Lie algebra: (a) skew anti-symmetry and (b) Jacobi identity.

the registration parameters. Because the inconsistency penalties require all registration parameter sets, all pairwise registrations must be performed simultaneously, which is undesirable for large-scale 3D mapping applications. In this paper, we will enforce the consistency constraints only locally to improve a single pairwise registration, with or without an augmented third supporting point cloud for the Jacobi identity constraint.

\section{CONSISTENT AND ROBUST ICP}

To increase the robustness of the point-to-plane registration, we propose two improvements to the standard ICP algorithm. The first problem is that sweeps do not fully overlap with each other or that one of the sweeps is incomplete (e.g. due to bad acquisition or objects/persons too close to the device blocking the line of sight). In [18], the authors introduce a maximum match distance parameter to reject point correspondences that are too far. Unfortunately, the choice of this parameter has a large impact on the convergence and accuracy. We propose a more symmetrical variant to solve this problem: we put an adaptive threshold on the match distance (between $\mathbf{p}_{i}$ and $\mathbf{q}_{i}^{\prime}$ ), which is computed as $x$ times the match distance of the dual correspondences (i.e. between $\mathbf{q}_{i}$ and $\mathbf{p}_{i}^{\prime}$ ). In the experiments we choose $x$ to be 1.25 , meaning that we tolerate that the match distance of $\mathbf{p}_{i}$ or $\mathbf{q}_{i}$ can be $25 \%$ larger than the minimum match distance of $\mathbf{p}_{i}$ and $\mathbf{q}_{i}$. The choice of $x$ in the range $] 1,2]$ will have a much lower impact on the convergence and accuracy than the maximum match distance parameter due to its adaptive behavior.

The second improvement is dealing with the $\ell_{2}$-norm in Equation (2), which is not robust to outliers. A well-known strategy is to adopt M-estimators [1], which is then typically solved using iterative re-weighted least squares (IRLS). In the experiments, we will use the Cauchy robust loss function $\rho(\mathrm{e})=\log \left(1+(\mathrm{e} / c)^{2}\right)$, with $c$ being a tuning parameter and e the residual error. Other robust loss functions such as the $\ell_{1}$-norm, Huber and Geman-McClure can be used as well.

Similar to [6], we will incorporate the consistency constraints as prior knowledge in a Bayesian maximum a posteriori framework. The new ICP metric is symmetric in $\mathbf{p}$ and $\mathbf{q}$ and combined with the skew anti-symmetry property, the metric becomes:

$$
\begin{aligned}
\hat{T}_{\mathbf{p q}}, \hat{T}_{\mathbf{q p}}=\underset{T_{\mathbf{p q}}, T_{\mathbf{q p}}}{\arg \min } \sum_{i=1}^{N_{\mathbf{p}}^{\prime}} \rho\left(\left(T_{\mathbf{p q}} \mathbf{p}_{i}-\mathbf{q}_{i}^{\prime}\right) \cdot \mathbf{n}_{\mathbf{q}_{i}^{\prime}}\right) \\
\quad+\sum_{i=1}^{N_{\mathbf{q}}^{\prime}} \rho\left(\left(T_{\mathbf{q p}} \mathbf{q}_{i}-\mathbf{p}_{i}^{\prime}\right) \cdot \mathbf{n}_{\mathbf{p}_{i}^{\prime}}\right) \\
+\lambda\left\|T_{\mathbf{p q}} T_{\mathbf{q p}}-I\right\|^{2}+\lambda\left\|T_{\mathbf{q p}} T_{\mathbf{p q}}-I\right\|^{2}
\end{aligned}
$$

where $\lambda$ is the regularization parameter and $N_{\mathbf{p}}^{\prime}$ and $N_{\mathbf{q}}^{\prime}$ are the number of points left after point correspondence rejection with the adaptive threshold. Both priors are mathematically seen the same, however, due to local minima in the solution space and the order of rotation axes that are applied in the 3D rotation matrix (which will have an impact on the accuracy of the floating point computations), it is better to incorporate both priors in the metric.

Note that the normals of both point clouds are incorporated in the metric, resulting in a plane-to-plane approach, similar to [18]. We opt for the sequential quadratic programming (SQP) algorithm to solve the non-linear minimization problem (3), instead of the standard IRLS approach. We refer the interested reader to [8] for more details on the SQP algorithm.

When we augment the pairwise registration with a third point cloud and include the Jacobi identity constraint, the metric becomes much more complex: instead of 4 terms, we will have to optimize 24 terms. The metric consists of 6 fidelity terms, 6 consistency terms for the skew antisymmetry constraint and 12 consistency terms for the Jacobian identity constraint. An example of the latter consistency term is $\lambda\left\|T_{\mathbf{q p}} T_{\mathbf{r q}} T_{\mathbf{p r}}-I\right\|^{2}$. We can see that adding a fourth, fifth, ... supporting point cloud will result in a tremendous increase of consistency terms, not to mention that even other Lie group structures can then be taken into account. This is also the main drawback of our proposed method compared to other ICP algorithms: the optimization problem is much larger and requires more computational power.

\section{EXPERIMENTAL RESULTS}

For the validation of ICP algorithms, we will use real LiDAR data that is obtained by a Velodyne High Definition LiDAR scanner (HDL-32e). The scanner has 32 lasers covering a vertical field-of-view (FOV) of 41.3 degrees hence resulting in a vertical resolution of 1.29 degrees. The head is continuously spinning at approximately $10 \mathrm{~Hz}$ covering a horizontal FOV of 360 degrees. The data set has been captured outdoors, obtained at a construction site, where the operator is also captured (which can be seen as clutter). Although IMU data has been collected, it is not a perfect way to generate ground truth data from it. Therefore, we will evaluate and validate ICP visually and objectively with back-projection errors. The backprojection error is denoted as the mean Euclidean distance of the reference point cloud and the same point cloud after forward and backward transformation. Ideally this would result in the identity transformation with zero back-projection error. 
We call our proposed methods rICP (robust ICP without consistency constraints), crICP (consistent and robust ICP as discussed in the previous section) and acrICP (consistent and robust ICP augmented with a third point cloud). We set the following parameters for our algorithms: $c=0.5,20$ iterations and $\lambda=\left(N_{\mathbf{p}}^{\prime}+N_{\mathbf{q}}^{\prime}\right) \cdot 10^{3}$ (because $\lambda$ heavily depends on the number of corresponding matches). We compare our algorithms with standard point-to-point ICP (denoted as pointICP), point-to-plane ICP (denoted as planeICP) and irlsICP [1] (with Tukey's biweight robust loss function). We set the outlier rejection on the correspondence matches on $40 \%$ for standard pointICP and planeICP [23].

In the first experiment, we compute the back-projection error where we first compute the transformation from $\mathbf{p}$ to $\mathbf{q}$ and back from $\mathbf{q}$ to $\mathbf{p}$. The error is then computed over all the points of $\mathbf{p}$. We have 50 runs where the sweeps are selected randomly, but with a large overlap (at least $80 \%$ coverage). The average results are shown in Table I.

TABLE I

AVERAGE BACK-PROJECTION ERROR $(\mu)$ AND STANDARD DEVIATION $(\sigma)$. THE BEST RESULT IS WRITTEN IN BOLD.

\begin{tabular}{lcc}
\hline Method & $\mu$ & $\sigma$ \\
\hline pointICP & 2.04 & 6.58 \\
planeICP & 74.98 & 301.84 \\
irlsICP & 25.54 & 31.37 \\
rICP & 7.28 & 11.50 \\
crICP & 0.94 & 1.26 \\
acrICP & $\mathbf{0 . 7 3}$ & $\mathbf{1 . 2 1}$ \\
\hline
\end{tabular}

We can observe that acrICP is the best performing ICP method, which is what we expect since the skew antisymmetry constraint is also optimized. crICP is performing slightly worse than acrICP because the supporting point cloud aids in having more consistent registrations (point clouds are also less sparse because there are more points available). However, this comes with a huge drawback: the computational load of acrICP is about 15 times higher than crICP (in MATLAB). The average back-projection error of pointICP is also relatively low because the metric (1) is symmetrical in $\mathbf{p}$ and $\mathbf{q}$ (although the correspondence matching is not completely symmetrical). Note that a low back-projection error does not necessarily means that the algorithm converges to the right solution. The large standard deviation for planeICP and irlsICP points to divergent registrations (i.e. where the consistency completely fails).

In the second experiment, we test the robustness of the ICP algorithm to incomplete point cloud. A part of the point cloud is missing because the operator was too close to the device resulting in non-measured data. The projection of the point clouds $\mathbf{p}$ and $\mathbf{q}$ on the ground plane is given in Figure 2.

The overlap after registration for different ICP algorithms is given in Figure 3. We can observe that pointICP and irlsICP completely diverge from the true solution, while planeICP

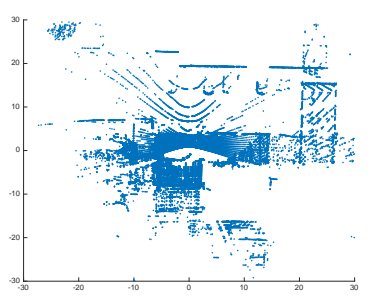

(a)

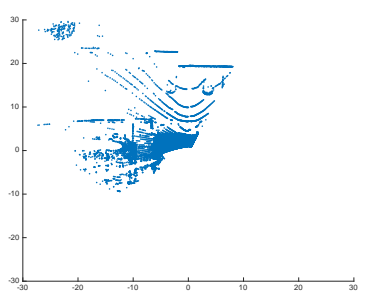

(b)
Fig. 2. Projected point clouds on the ground plane: (a) $\mathbf{p}$ and (b) $\mathbf{q}$.

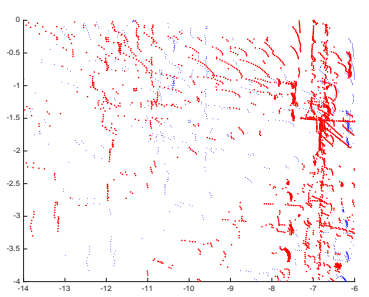

(a) pointICP

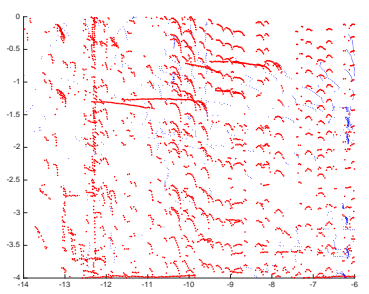

(c) irlsICP

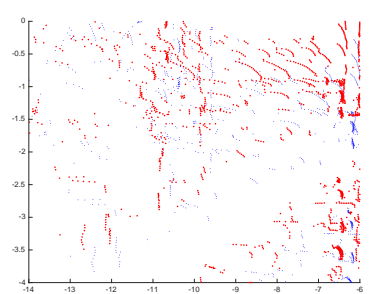

(b) planeICP

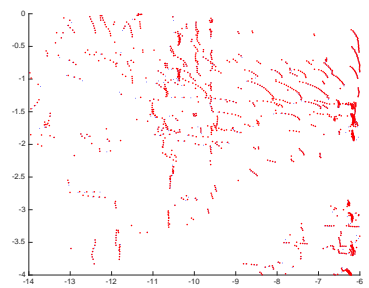

(d) $\mathrm{rICP}$

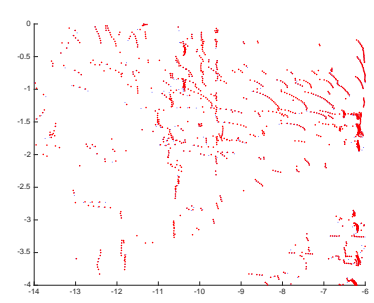

(e) $\operatorname{crICP}$

Fig. 3. Detailed part of the overlap between transformed $\mathbf{p}$ and $\mathbf{q}$.

clearly is closer to the solution, but still does not give the correct solution. The proposed methods rICP and crICP converges to the right solution. The proposed adaptive threshold on the match distances prevents that the estimation is biased, while outlier rejection based on a fixed percentage or fixed threshold will not cope completely with incomplete point clouds.

In the third experiment, we build a complete reconstruction by successive pairwise point cloud registrations. The complete reconstruction is given in Figure 4. A part of the 3D mappings of the construction site for planeICP, rICP and crICP are given in Figure 5.

We notice that our proposed methods are more accurate 


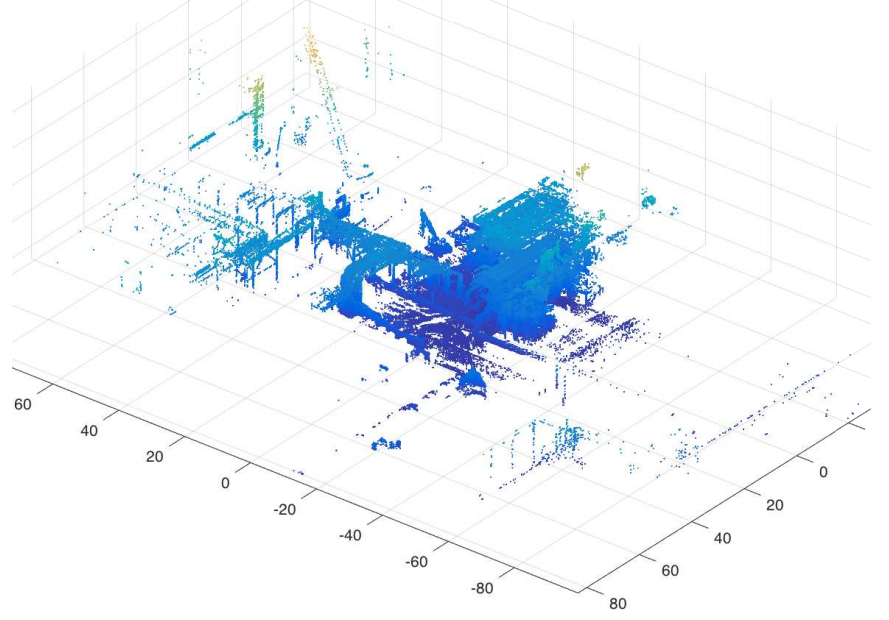

Fig. 4. Complete 3D mapping of the reconstruction site.

than planeICP, which is clearly visible due to the errors on the reconstruction of the wall on the left of the image. rICP and crICP produce similar visual results, but crICP is more consistent.

\section{CONCLUSION}

For the registration of sparse and inhomogeneous point clouds, we have introduced a novel consistent and robust ICP algorithm. We improve the ICP robustness by incorporating an adaptive threshold on the match distance of the point correspondences and by adopting M-estimators in the pointto-plane metric. Next, we improve the ICP consistency by enforcing valid Lie group structures such as the skew antisymmetry and the Jacobi identity to the different transformations in a Bayesian framework. Experimental results show that the proposed method is more consistent and accurate for real Velodyne LiDAR data compared to other ICP algorithms. Future work is to include the proposed ICP algorithm in more advanced LiDAR SLAM systems and to accelerate the algorithm using GPU-computing, using for example the Quasar programming framework [9].

\section{ACKNOWLEDGMENT}

This research was carried out as part of the iMinds ICON GiPA project (https://www.iminds.be/en/projects/2014/03/20/ gipa). The work was made possible by a cooperative agreement that was administered by ir. Pieter Raes, project manager at Dow Benelux BV.

\section{REFERENCES}

[1] Bergström, P., and Edlund, O. Robust registration of point sets using iteratively reweighted least squares, Computational Optimization and Applications, vol. 58, no. 3, pp. 543-561, 2014.

[2] Besl, P.J., McKay, H.D., A method for registration of 3-D shapes, IEEE Trans. Pattern Anal. Machine Intell., vol. 14, no. 2, pp. 239-256, 1992.

[3] Chen, Y., Medioni, G., Object modelling by registration of multiple range images, Image Vision Computing, vol. 10, no. 3, pp. 145-155, 1992.

[4] Du, S., Zheng, N., Ying, S., and Liu, J., Affine iterative closest point algorithm for point set registration, Pattern Recognition Letters, vol. 31, no. 9, pp. 791-799, 2010.

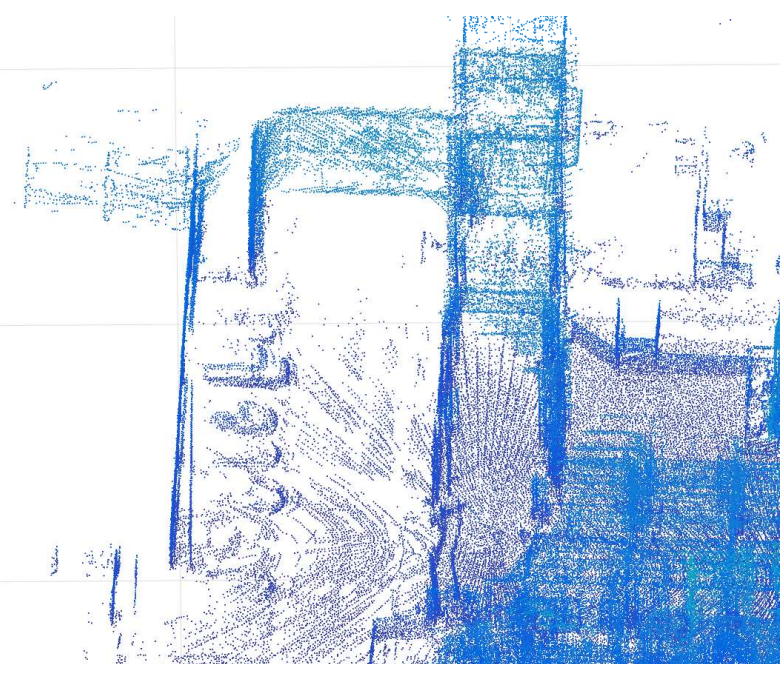

(a) planeICP

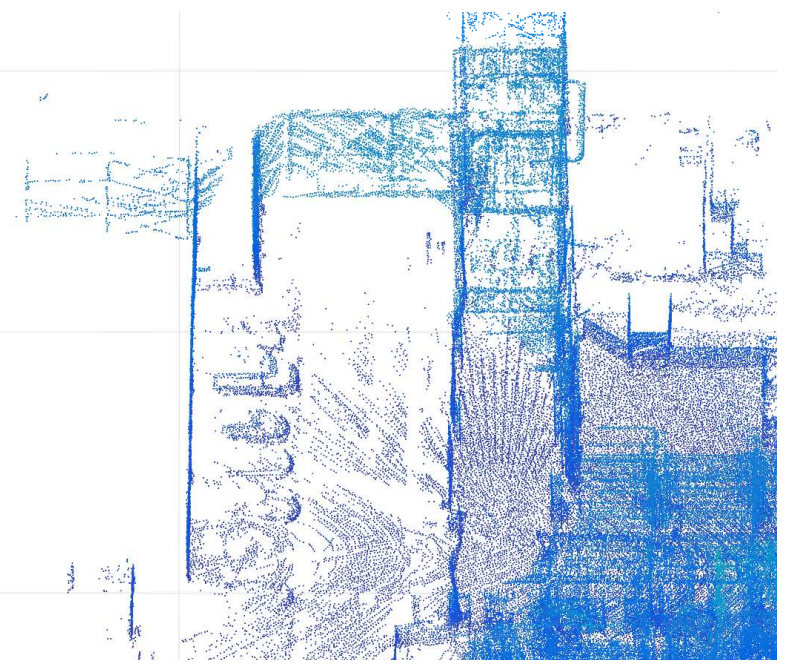

(b) $\mathrm{rICP}$

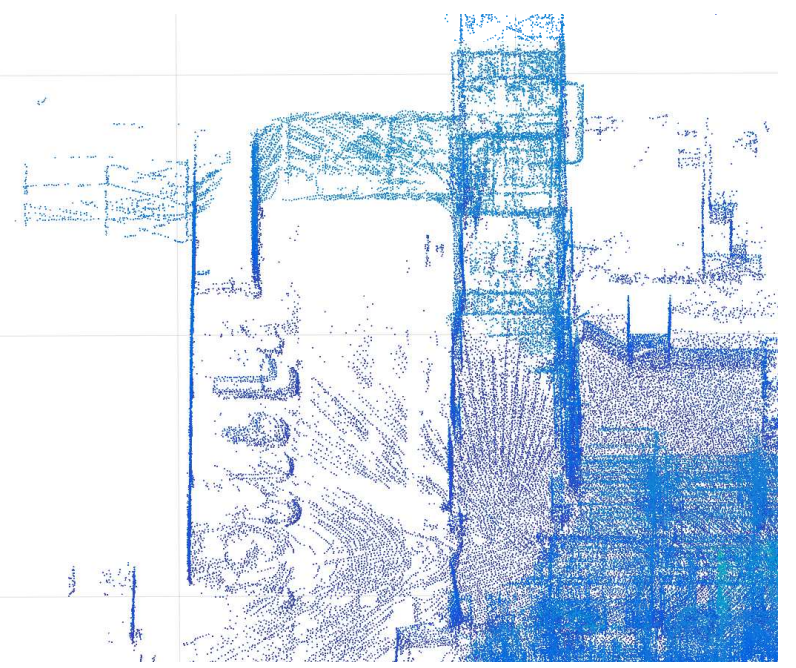

(c) $\mathrm{crICP}$

Fig. 5. Detailed part of the 3D mapping. 
[5] Einicke, G.A., Smoothing, Filtering and Prediction Estimating The Past, Pre-sent and Future, InTech, ISBN 978-953-307-752-9, 2012.

[6] Farsiu, S., Elad, M., and Milanfar, P., Constrained, globally optimal, multi-frame motion estimation, In Proceedings of IEEE Workshop on Statistical Signal Processing, pp. 1396-1401, 2005.

[7] Fitzgibbon, A.W., Robust registration of $2 D$ and $3 D$ point sets, Image Vision Computing, vol. 21, no. 1314, pp. 1145-1153, 2003.

[8] Gill, P.E., Murray, W., and Wright, M.H., Numerical Linear Algebra and Optimization, vol. 1, Addison Wesley, 1991.

[9] Goossens, B., De Vylder, J., and Philips, W., Quasar - a New Heterogeneous Programming Framework for Image and Video Processing Algorithms on CPU and GPU, IEEE Int. Conf. on Image Processing (ICIP), pp. 2183-2185, 2014.

[10] Govindu, V., Lie-algebraic averaging for globally consistent motion estimation, In Proceedings of the International Conference on Computer Vision and Pattern Recognition (CVPR), vol 1, pp. 684-691, 2004.

[11] Jost, T., Hugli, H., Multi-resolution ICP with heuristic closest point search for fast and robust $3 D$ registration of range images, In Proc. Fourth International Conference on 3-D Digital Imaging and Modeling (3DIM), pp. 427-433, 2003.

[12] Makadia, A., Patterson, A.I., Daniilidis, K., Fully automatic registration of $3 D$ point clouds, In Proc. 2006 IEEE Computer Society Conf. on Computer Vision and Pattern Recognition (CVPR), pp. 1297-1304, 2006.

[13] Moosmann, F., Stiller, C., Velodyne SLAM, In Proceedings of the IEEE Intelligent Vehicles Symposium, pp. 393-398, 2011.

[14] Petrie, G., Mobile Mapping Systems: An Introduction to the Technology, GeoIn-formatics, vol. 13, no. 1, pp. 32-43, 2010.

[15] Planitz, B.M., Maeder, A.J., Williams, J.A., The correspondence framework for $3 D$ surface matching algorithms, Computer Vision and Image Understanding, vol. 97, no. 3, pp. 347-383, 2005.

[16] Pomerleau, F., Colas, F., Siegwart, R., and Magnenat, S., Comparing ICP variants on real-world data sets, Autonomous Robots, vol. 34, no. 3, pp. 133-148, 2013.

[17] Puente, I., Gonzales-Jorge, H., Martinez-Sanchez, J., and Arias, P. Review of Mobile Mapping and Surveying Technologies, Measurement, vol. 46, pp. 2127-2145, 2013.

[18] Segal, A.V., Haehnel, D., and Thrun, S., Generalized-ICP, In Robotics: Science and Systems, 2009.

[19] Smith, E.R., King, B.J., Stewart, C.V., Radke, R.J., Registration of com bined range-intensity scans: Initialization through verification, Computer Vision and Image Understanding, vol. 110, no. 2, pp. 226-244, 2008.

[20] Sprickerhof, J., Nüchter, A., Lingemann, K., Hertzberg, J., An explicit loop closing technique for $6 D$ slam, In Proceedings of KoREMA, pp. 229-234, 2009.

[21] Triggs, B., McLauchlan, P., Hartley, R., and Fitzgibbon, A., Bundle adjustment - a modern synthesis, In Proceedings of the International Workshop on Vision Algorithms: Theory and Practice, Lecture Notes in Computer Science, Springer-Verlag, vol. 1883, pp. 298-372, 1999.

[22] Yen, K.S., Ravani, B., and Lasky, T.Y., LiDAR for Data Efficiency, tech. report WA-RD 778.1, University of California, 2011.

[23] Zhang, Z., Iterative point matching for registration of free-form curves and surfaces, International Journal on Computer Vision, vol. 13, no. 2, pp. 119-152, 1994.

[24] Zhang, J., Singh, S., LOAM: Lidar odometry and mapping in realtime, In Robotics: Science and Systems Conference (RSS), 2014.

[25] Zhang, J., Singh, S., Visual-lidar Odometry and Mapping: Low-drift, Robust, and Fast, In Proceedings of IEEE International Conference on Robotics and Automation (ICRA), pp. 2174-2181, 2015. 